\title{
三维有序大孔 $\mathrm{Al}_{2} \mathbf{O}_{3}$ 制备的新方法及表征 *
}

\author{
邬泉周沈勇李玉光 \\ (中山大学化学与化学工程学院, 广州 510275)
}

\begin{abstract}
摘要以聚苯乙烯胶晶为模板, 用 $\mathrm{Al}\left(\mathrm{NO}_{3}\right)_{3} \cdot 9 \mathrm{H}_{2} \mathrm{O}$ 为前驱物, 使用柠檬酸为配体, 成功地制备了孔径为 $250 \sim$ $350 \mathrm{~nm}$ 的三维有序大孔 $\mathrm{Al}_{2} \mathrm{O}_{3}$ 材料. SEM 观察表明, 所得大孔材料孔结构规则排列, 孔与孔之间通过小孔相连, 形成了一个三维有序排列的蜂窝状结构. 实验发现, 以 $\mathrm{Al}\left(\mathrm{NO}_{3}\right)_{3} \cdot 9 \mathrm{H}_{2} \mathrm{O}$ 为前驱物, 加人柠檬酸可以防止团聚粒 子的产生, 有利于三维有序结构的形成. 前驱物浓度在 $0.5 \sim 0.8 \mathrm{~mol} \cdot \mathrm{L}^{-1}$ 范围内均能得到较好的三维有序大 孔结构. 在 $1100{ }^{\circ} \mathrm{C}$ 焙烧 $2 \mathrm{~h}$ 后, $\mathrm{Al}_{2} \mathrm{O}_{3}$ 大孔材料仍能保持完整的规则孔结构特征, 表现出较高的热稳定性.
\end{abstract}

关键词: 三维有序大孔, $\mathrm{Al}_{2} \mathrm{O}_{3}$, 聚苯乙烯胶晶 中图分类号: 0643

大孔 ( $\geqslant 50 \mathrm{~nm})$ 金属氧化物作为过滤分离材 料、催化剂载体、色谱载体、细胞固定载体等在工业 上应用广泛。许多化学化工过程和分离过程, 常常 通过材料孔尺寸控制或扩散控制达到分子水平的 篮分效应。但传统制备工艺很难控制大孔结构,得 到的大孔材料孔径分布比较宽 ${ }^{[1]}$. 上世纪九十年代 末, Imhof 和 Velev 等 ${ }^{[2-3]}$ 分别用微乳法和胶晶模板 法成功地制备了有序的大孔材料, 引起广泛注意. 尤其是用胶晶模板法制备三维有序大孔材料, 不仅 具有一般大孔材料的物理化学特性, 而且因为它孔 径分布窄, 孔道排列整齐有序, 用作分离及载体材 料更具有选择性. 如果材料孔径控制在可见光波 长范围, 在光学上用作光子晶体有着诱人的应用前 景. 因此, 这种模板法被认为是制备三维有序大孔 材料最有效的一种方法. 近几年来, 对三维有序大 孔材料的研究备受人们的关注. 胶晶模板法主要 按以下三步进行: 1) 制备有序排列的胶晶模板;2) 往模板间隙内填充所需目标产物的前驱物; 3) 去 掉模板得到产物. 现在使用最多的模板胶晶是 聚苯乙烯 $(\mathrm{PS})$ 胶晶 ${ }^{[3-6]} 、 \mathrm{SiO}_{2}$ 胶晶 ${ }^{[7]}$ 和 PMMA (polymethyl methacrylate) 胶晶 ${ }^{[8]}$. 由于大孔材料的 形成是在模板间隙内发生的流体 - 固体转变过程, 因此, 能否制备出理想的三维有序大孔产物, 与前 驱物的物理化学特性、浓度、流动性和操作条件等
因素有关. 目前, 制备三维大孔材料新方法仍在不 断开发, 原料选择、配比和实验条件仍在不断改进 和探索中.

$\mathrm{Al}_{2} \mathrm{O}_{3}$ 是工业上广泛使用的干燥剂、吸附剂、催 化剂、催化剂载体及分离材料, 将 $\mathrm{Al}_{2} \mathrm{O}_{3}$ 制成三维有 序的大孔材料是十分有意义的课题. Bolland 等 ${ }^{[4]}$ 用 聚苯乙烯胶晶为模板, 用三仲丁醇铝为前驱物制备 了具有三维有序大孔结构的 $\mathrm{Al}_{2} \mathrm{O}_{3}$ 材料. 但用醇盐 为铝源, 价格高, 且易受环境湿度的影响, 操作条件 要求苛刻. Lei 等 ${ }^{[9]}$ 以 $\mathrm{AlCl}_{3}$ 为前驱物, 用二次模板 法制备了有序的大孔 $\mathrm{Al}_{2} \mathrm{O}_{3}$, 即先以 $\mathrm{SiO}_{2}$ 胶晶为模 板, 制备出有序大孔碳材料, 然后利用大孔碳为模 板制备大孔 $\mathrm{Al}_{2} \mathrm{O}_{3}$ 材料. 这种方法虽然原料比较便 宜, 但制备过程比较复杂, 需要经过一次模板转换, 而且中间要用 $\mathrm{HF}$ 去除 $\mathrm{SiO}_{2}$ 模板.

本文以聚苯乙烯胶晶为模板, 采用廉价的 $\mathrm{Al}\left(\mathrm{NO}_{3}\right)_{3} \cdot 9 \mathrm{H}_{2} \mathrm{O}$ 为铝源, 并用柠檬酸为鳌合剂, 改善 大孔骨架形成过程, 成功地制备出了具有三维有序 大孔结构的 $\mathrm{Al}_{2} \mathrm{O}_{3}$ 材料, 且其具有很高的热稳定性.

\section{1 实验部分}

\section{1 试 剂}

$\mathrm{Al}\left(\mathrm{NO}_{3}\right)_{3} \cdot 9 \mathrm{H}_{2} \mathrm{O}$ 、柠檬酸、乙醇 $(95 \%)$ 均为 $\mathrm{AR}$ 级试剂。

2003-02-17 收到初稿, 2003-04-02 收到修改稿. 联系人: 李玉光 (E-mail: ceslyg @ zsu. edu. cn; Tel: 020-84039647). *广东省科技厅 “五”重点专项 (A3040302) 资助项目 

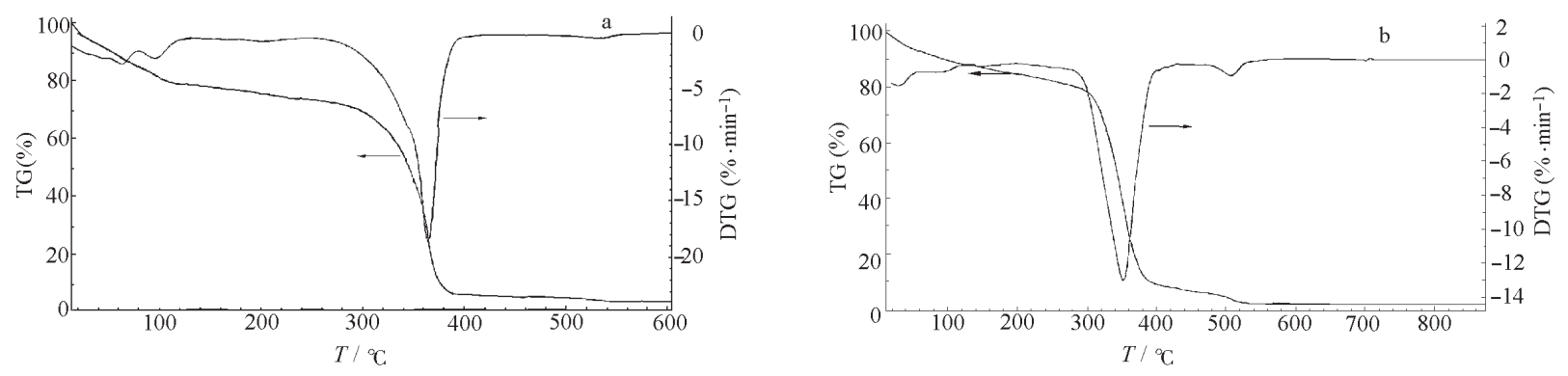

图 1 升温速率为 $10{ }^{\circ} \mathrm{C} \cdot \mathrm{min}^{-1}$ 的样品热重曲线

Fig. 1 TG curves of the samples at heating rate of $10{ }^{\circ} \mathrm{C} \cdot \mathrm{min}^{-1}$

(a) sample A; (b) sample B

\section{2 三维有序大孔 $\mathrm{Al}_{2} \mathrm{O}_{3}$ 的制备及表征}

直径在 $600 \mathrm{~nm}$ 左右的聚苯乙烯微球胶晶按文 献制备方法得到 ${ }^{[6]}$.

前驱物溶液按是否加人柠檬酸配置成两种溶 液: 将 $0.01 \mathrm{~mol} \mathrm{Al}\left(\mathrm{NO}_{3}\right)_{3} \cdot 9 \mathrm{H}_{2} \mathrm{O}$ 溶于 $10 \mathrm{~mL}$ 乙醇 中, 搅拌溶解得无色透明溶液 (I); 将 $\mathrm{Al}\left(\mathrm{NO}_{3}\right)_{3}$. $9 \mathrm{H}_{2} \mathrm{O}$ 和柠檬酸按等摩尔比混合后, 溶于乙醇, 配制 成浓度为 $0.1 、 0.5 、 0.7$ 和 $0.8 \mathrm{~mol} \cdot \mathrm{L}^{-1}$ 的前驱物溶 液 (II), 将一定量的聚苯乙烯胶晶放置在布氏漏斗 上, 在抽滤下将溶液滴加到胶晶上, 让其充分渗透进 微球的间隙内, 然后在 $60 \sim 70{ }^{\circ} \mathrm{C}$ 下干燥 $1 \sim 2 \mathrm{~h}$, 重 复数次, 得干燥样品. 将所得样品置于管式炉内, 在 空气气氛下缓慢升温至 $300{ }^{\circ} \mathrm{C}$, 恒温 $5 \mathrm{~h}$ 以去掉聚 苯乙烯模板, 然后再升至一定温度进行焙烧, 最后得 $\mathrm{Al}_{2} \mathrm{O}_{3}$ 固体。

样品孔结构特征用日本电子 JSM-6330F 型场 发射扫描电子显微镜和日本 Hitachi 公司 S-520 扫 描电子显微镜表征, 晶相分析是用日本理学电机 $\mathrm{D} / \mathrm{Max}-\mathrm{IIIAX}$ 射线 (粉末) 衍射仪 (XRD) 测定, $\mathrm{TG}$ 测定使用德国 Netzsch 公司 TG-209 热重分析仪, TG-IR 分析用德国 Netzsch 公司 TG-209 和 Bruker 公司 Victor ${ }^{\mathrm{TM}}-22$ 红外光谱联用仪, 积碳含量由德国 Elementar 公司的 Vario EL CHNS-O 元素分析仪测 定.

\section{2 结果与讨论}

\section{1 热重及热重 - 红外分析}

TG 及 TG-IR 分析是在空气气氛下, 在 $10{ }^{\circ} \mathrm{C} \cdot$ $\mathrm{min}^{-1}$ 升温速率的条件下进行。图 $1(\mathrm{a})$ 和 $1(\mathrm{~b})$ 分 别是用同体积摩尔浓度的溶液 (I) 和溶液 (II) 滴加 3 次所制备的样品 $\mathrm{A}$ 和 $\mathrm{B}$ 的热重曲线. 可以看到, 在 $280{ }^{\circ} \mathrm{C}$ 前是缓慢失重, 失重率大约 $20 \%(w)$, 主要是 表面水、结构水、溶剂、 $\mathrm{HNO}_{3}$ 和硝酸根分解产物
$\left(\mathrm{NO}_{2} 、 \mathrm{~N}_{2} \mathrm{O} \text { 和 } \mathrm{NO}\right)^{[10]}$ 的挥发和未配位柠檬酸的氧化 分解; $280 \sim 390{ }^{\circ} \mathrm{C}$ 快速失重是 PS 的氧化分解, 失重 率约为 $70 \%$. 在 $390{ }^{\circ} \mathrm{C}$ 时, 样品 $\mathrm{A}$ 和样品 $\mathrm{B}$ 的残留 量各为 $5.84 \%$ 和 $10.7 \%(w)$, 表明样品 B 残留量较 高; $390{ }^{\circ} \mathrm{C}$ 以后, 样品 $\mathrm{B}$ 失重加快. 两者热重曲线最 显著的差别是: 样品 $\mathrm{B}$ 在 $458 \sim 520{ }^{\circ} \mathrm{C}$ 有一个显著 的失重峰, 失重率达 $2.6 \%(w)$.

对前驱物溶液 (II), 经 $60{ }^{\circ} \mathrm{C}$ 烘干后进行了 TG-IR 分析, 图 2 是 TG-IR 分析结果, 可以看到, 在 $480{ }^{\circ} \mathrm{C}$ 附近, IR 检测到很强的 $\mathrm{CO}_{2}$ 吸收峰, 这说明 失重由柠檬酸铝的氧化分解所至。陈忠等 ${ }^{[11]}$ 在用 柠檬酸铝制备纳米 $\mathrm{Al}_{2} \mathrm{O}_{3}$ 的研究中, 用 DTA 分析观 察到在 $473{ }^{\circ} \mathrm{C}$ 处有类似的放热峰. $550{ }^{\circ} \mathrm{C}$ 以后, 两个 样品已基本恒重.

\section{2 柠檬酸对形成三维大孔结构的影响}

图 3(a) 是样品 $\mathrm{A}$ 经 $600{ }^{\circ} \mathrm{C}$ 焙烧 $8 \mathrm{~h}$ 后所得产 物的 SEM 图. 由图可见, 用溶液 (I) 制得的 $\mathrm{Al}_{2} \mathrm{O}_{3}$ 材料能得到大孔骨架, 但三维骨架结构较差, 在孔结 构破裂处发现有圆形及椭圆形的团聚粒子。热重分 析表明, $550{ }^{\circ} \mathrm{C}$ 焙烧后样品已基本恒重, 说明那些团 聚粒子为 $\mathrm{Al}_{2} \mathrm{O}_{3}$ 团聚体. 这些团聚粒子的确切形成 过程还不很清楚, 可能与 $\mathrm{Al}\left(\mathrm{NO}_{3}\right)_{3} \cdot 9 \mathrm{H}_{2} \mathrm{O}$ 热分解

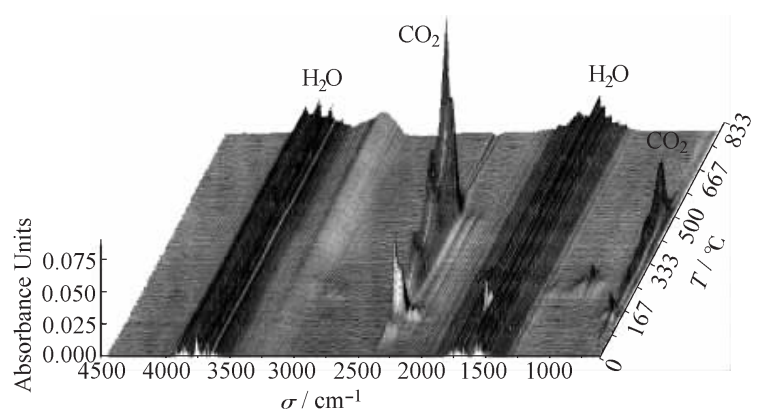

图 2 由前驱物溶液 (II) 所得样品的 TG-IR 谱图

Fig. 2 TG-IR image of the sample obtained from the precursorsolution (II) 

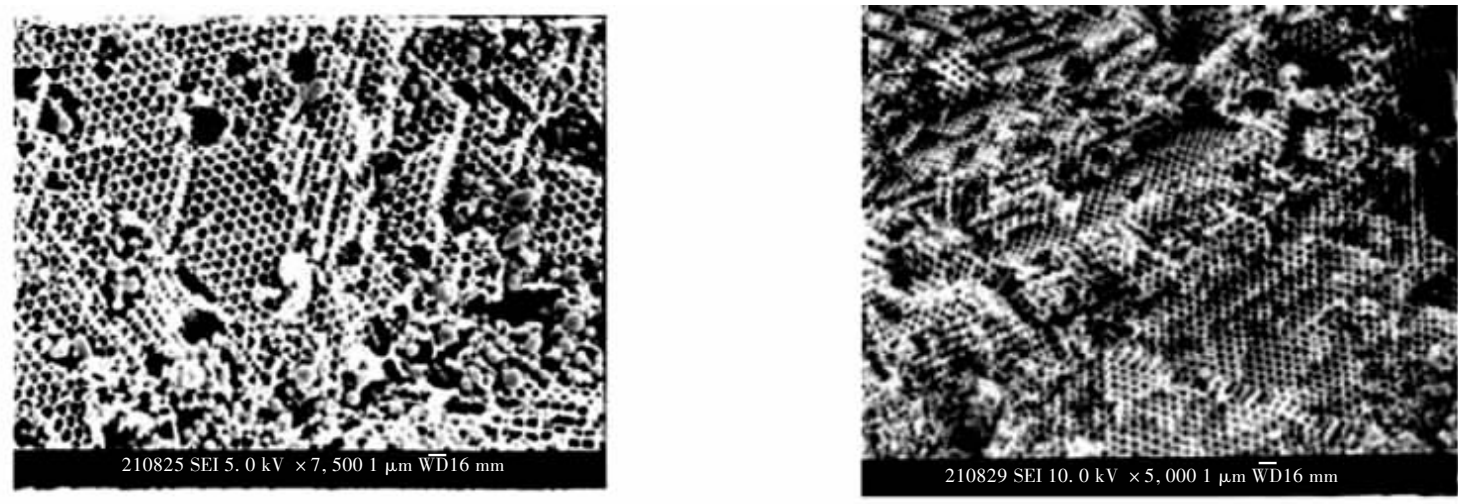

图 3 分别用同浓度前驱物溶液(I)和 (II) 制备的大孔 $\mathrm{Al}_{2} \mathrm{O}_{3}$ 的 SEM 图

Fig. 3 SEM images of the macroporous $\mathrm{Al}_{2} \mathrm{O}_{3}$ obtained by using different precursor solution (I) and (II) for the same concentration

(a) solution (I); (b) solution (II)

过程密切相关. $\mathrm{Al}\left(\mathrm{NO}_{3}\right)_{3} \cdot 9 \mathrm{H}_{2} \mathrm{O}$ 在 $73.5{ }^{\circ} \mathrm{C}$ 熔 化, 加热至 $130 \sim 135{ }^{\circ} \mathrm{C}$, 分解为硝酸和碱式硝酸 铝 $\mathrm{Al}(\mathrm{OH})_{x}\left(\mathrm{NO}_{3}\right)_{y}(x+y=3)$, 加热至 $500{ }^{\circ} \mathrm{C}$ 以上 分解为 $\mathrm{Al}_{2} \mathrm{O}_{3}$ 和氮的氧化物 ${ }^{[12]}$. Pacewska 等 ${ }^{[10]}$ 用 TG、DTA、MS、IR 等技术对 $\mathrm{Al}\left(\mathrm{NO}_{3}\right)_{3} \cdot 9 \mathrm{H}_{2} \mathrm{O}$ 热分 解过程进行了详细研究, 发现这一过程中伴随着熔 化及一系列的脱水、脱硝酸根、水解和脱差基过程. 在 $170{ }^{\circ} \mathrm{C}$ 以上即发生由水解产生的碱式硝酸铝脱羟 基化作用, 从 IR 谱可观察到 $200{ }^{\circ} \mathrm{C}$ 处理后的样品有 显著 $\mathrm{NO}_{3}^{-}$吸收峰, $400{ }^{\circ} \mathrm{C}$ 处理后, 仍可检测到这个吸 收峰. 可以推测, 在 $300{ }^{\circ} \mathrm{C}$ 时, PS 氧化分解生成大 量水, 碱式硝酸铝进一步水解。在后续热处理中, 带 羟基的小粒子间脱羟基化聚合, 并可能进一步烧结 形成较大球型的粒子.

图 3(b) 是样品 $\mathrm{B}$ 经 $800{ }^{\circ} \mathrm{C}$ 焙烧 $8 \mathrm{~h}$ 后所得产 物的 SEM 图. 可以观察到, 经高温长时间焙烧, 产
物仍具有良好的三维大孔骨架, 并在大面积范围内 都未发现有团聚粒子. 说明柠檬酸的加人改善了 $\mathrm{Al}_{2} \mathrm{O}_{3}$ 三维大孔结构形成过程. 柠檬酸是一种含硬 碱 $\mathrm{O}^{-}$的配位体, 能和 “硬酸” $\mathrm{Al}^{3+}$ 离子形成稳定的 柠檬酸铝螯合物。柠檬酸含有四个供氧基, 有多 种配位可能性, NMR 研究已经表明, 在酸性 $(\mathrm{pH}<$ 3 ) 条件下, 主要以单核络合物 $\left[\mathrm{Al}\left(\mathrm{C}_{6} \mathrm{H}_{6} \mathrm{O}_{7}\right)\right]^{+}$和 $\mathrm{Al}\left(\mathrm{C}_{6} \mathrm{H}_{5} \mathrm{O}_{7}\right)$ 存在, 在 $\mathrm{pH}=4 \sim 9$ 时, 以热力学最稳定 的三聚体形式存在 ${ }^{[13]}$. 本工作的 $\mathrm{TG}$ 和 TG-IR 结 果表明, 柠檬酸铝直至 $458{ }^{\circ} \mathrm{C}$ 才开始氧化分解. 这 意味着, 在 $300{ }^{\circ} \mathrm{C} \mathrm{PS}$ 氧化分解过程中, 柠檬酸铝鳌 合物未发生分解, 仍然保留原来的状态。陈忠等 ${ }^{[11]}$ 利用柠檬酸铝的特性, 制备出纳米 $\mathrm{Al}_{2} \mathrm{O}_{3}$. 本工作进 一步表明, 加入柠檬酸避免粒子团聚, 有利于三维规 则排列大孔结构的形成. 本文以下所制备的三维有 序大孔 $\mathrm{Al}_{2} \mathrm{O}_{3}$ 样品均选择溶液 (II) 为前驱物溶液.
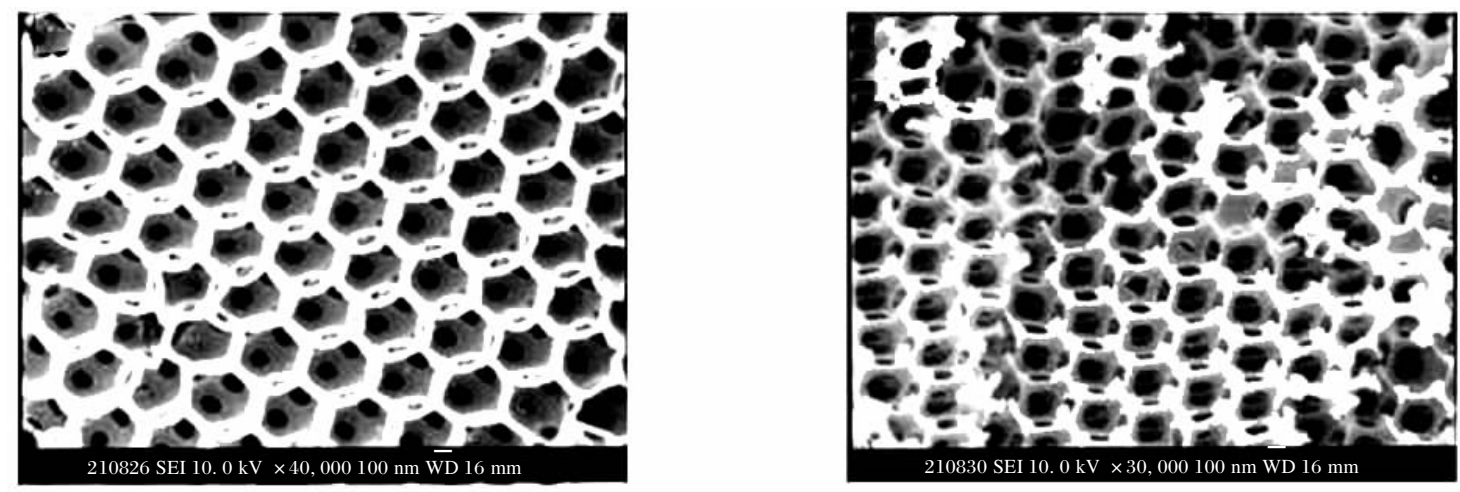

图 4 大孔 $\mathrm{Al}_{2} \mathrm{O}_{3}$ 的 $\mathrm{SEM}$ 图

Fig. 4 SEM images of macroporous $\mathrm{Al}_{2} \mathrm{O}_{3}$

(a) $0.7 \mathrm{~mol} \cdot \mathrm{L}^{-1}$ precursor, dropped 3 times, calcined at $800{ }^{\circ} \mathrm{C}$ for $8 \mathrm{~h}$; (b) $0.8 \mathrm{~mol} \cdot \mathrm{L}^{-1}$ precursor, dropped 3 times, calcined at $800{ }^{\circ} \mathrm{C}$ for $8 \mathrm{~h}$ 
表 1 不同制备参数对大孔结构的影响

Table 1. Effect of the preparation parameters on the structure of the macroporous $\mathrm{Al}_{2} \mathrm{O}_{3}$ materials

\begin{tabular}{|c|c|c|c|c|c|c|c|}
\hline No. & $\frac{c}{\mathrm{~mol} \cdot \mathrm{L}^{-1}}$ & Drop time (time) & $T_{\text {calcinations }} /{ }^{\circ} \mathrm{C}$ & $t_{\text {calcinations }} / \mathrm{h}$ & $d_{\text {pore }} / \mathrm{nm}$ & Shrinkage extent $(\%)$ & XRD phase \\
\hline Sample 1 & 0.1 & 1 & 600 & 8 & - & - & Amorphous \\
\hline Sample 2 & 0.1 & 3 & 600 & 8 & $\sim 290$ & $\sim 52$ & Amorphous \\
\hline Sample 3 & 0.5 & 3 & 600 & 8 & $250 \sim 310$ & $48 \sim 58$ & Amorphous \\
\hline Sample 4 & 0.5 & 3 & 800 & 8 & $\sim 250$ & $\sim 58$ & $\gamma-\mathrm{Al}_{2} \mathrm{O}_{3}$ \\
\hline Sample 5 & 0.7 & 3 & 800 & 8 & $\sim 340$ & $\sim 44$ & $\gamma-\mathrm{Al}_{2} \mathrm{O}_{3}$ \\
\hline Sample 6 & 0.8 & 3 & 800 & 8 & $\sim 350$ & $\sim 42$ & $\gamma-\mathrm{Al}_{2} \mathrm{O}_{3}$ \\
\hline Sample 7 & 0.8 & 3 & 1100 & 2 & $270 \sim 300$ & $50 \sim 55$ & $\delta, \theta-\mathrm{Al}_{2} \mathrm{O}_{3}$ \\
\hline Sample 8 & 0.8 & 3 & 1200 & 2 & - & - & $\theta-\mathrm{Al}_{2} \mathrm{O}_{3}$ \\
\hline
\end{tabular}

\section{3 前驱物浓度对形成三维大孔结构的影响}

前驱物溶液浓度对形成三维大孔结构是重要影 响因素之一。实验结果表明, 以 $0.1 \mathrm{~mol} \cdot \mathrm{L}^{-1}$ 的前 驱物溶液浓度滴加一次, 前驱物在模板间隙中沉积 量太少, 不足以形成大孔结构骨架. 增加滴加次数 可以弥补浓度低的不足, 如滴加三次, 能形成大孔骨 架, 但仍未能形成大尺度的三维有序结构. 增大沉 积量的另一有效方法是增大前驱物溶液的浓度. 本 研究表明, 前驱物浓度在 $0.5 \sim 0.8 \mathrm{~mol} \cdot \mathrm{L}^{-1}$ 范围 能得到三维有序的大孔结构。

图 4(a) 和 (b) 分别是浓度为 $0.7 \mathrm{~mol} \cdot \mathrm{L}^{-1}$ 和 $0.8 \mathrm{~mol} \cdot \mathrm{L}^{-1}$ 的前驱物滴加 3 次所得样品的 SEM 照片 . 由照片可见, 大孔孔结构规则排列, 孔与孔之 间有小孔相通, 形成了一种三维有序的蜂窝状结构, 此结构可认为是具有面心立方结构的聚苯乙烯胶晶 的翻版, 原来胶晶的位置变为空腔. 图 4(a) 是面心 立方结构的 (111) 面, 图 4(b) 是面心立方结构的 (100) 面. 可以看出, 空腔通过小孔相连, 形成纵横 交错孔道。图 4(b)显示, 孔结构不甚完整, 孔壁厚 度不很均匀, 这可能是浓度偏高, 粘度较大, 滴加过 程容易发生局部积聚, 较难在模板间隙内达到均匀 分布的缘故. 从 SEM 图中还测出孔壁的厚度约在 $37 \sim 50 \mathrm{~nm}$ 之间.

比较所得大孔材料的孔径 (相邻大孔中心与中 心间的距离)与模板聚苯乙烯微球的直径可以发现, 不同浓度所制备样品的孔径有不同程度的收缩, 如 表 1 所示. 前驱物浓度在 $0.1 \sim 0.5 \mathrm{~mol} \cdot \mathrm{L}^{-1}$ 内, 孔径收缩约为 $48 \% \sim 58 \%$, 前驱物浓度为 0.8 $\mathrm{mol} \cdot \mathrm{L}^{-1}$ 时, 孔径收缩约为 $42 \%$. 说明在一定范围 内增大前驱物的浓度可以减小收缩率, 因为高浓度 前驱物在模板间隙内沉积量比低浓度大。比较文献

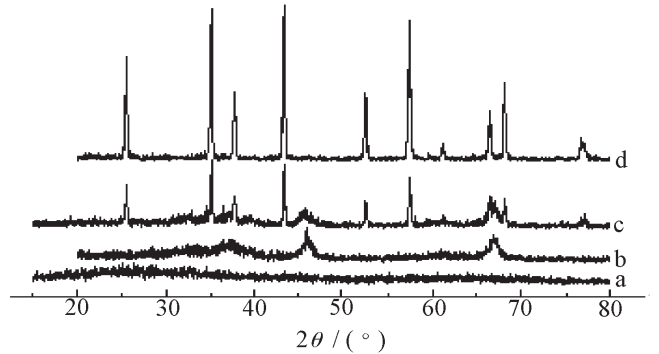

图 5 大孔 $\mathrm{Al}_{2} \mathrm{O}_{3} \mathrm{XRD}$ 晶相分析图谱

Fig. 5 Powder XRD patterns of the macroporous $\mathrm{Al}_{2} \mathrm{O}_{3}$

(a) and (b) calcined at $600{ }^{\circ} \mathrm{C}$ and $800{ }^{\circ} \mathrm{C}$ for $8 \mathrm{~h}$, respectively; (c) and (d) calcined at $1100{ }^{\circ} \mathrm{C}$ and $1200{ }^{\circ} \mathrm{C}$ for $2 \mathrm{~h}$, respectively

结果, 我们样品的孔径收缩率明显比用金属醇盐溶 胶 - 凝胶法制备的样品大一些 ${ }^{[4]}$, 但是, 与 Zhang 等 ${ }^{[14]}$ 用 EDTA 作为配体所制备的稀土大孔材料的 孔径收缩率相当, 说明不同分子大小的配体热分解 对孔径收缩有显著影响.

\section{4 焙烧温度对孔结构和 $\mathrm{Al}_{2} \mathrm{O}_{3}$ 晶型的影响}

焙烧目的在于去掉聚苯乙烯模板, 同时使柠檬 酸铝转化成具有一定晶型的氧化铝. 升温速率过 大, 将产生大量气泡不利于规则大孔的形成 ${ }^{[5]}$, 本实 验选择升温速率 $<5{ }^{\circ} \mathrm{C} \cdot \mathrm{min}^{-1}$, 并根据热重分析结 果, 在 $300{ }^{\circ} \mathrm{C}$ 时恒温 $5 \mathrm{~h}$, 以缓慢燃烧去掉聚苯乙烯 模板. 样品经 $600{ }^{\circ} \mathrm{C}$ 进一步焙烧 $8 \mathrm{~h}$ 后, 只有痕量 积碳检出 $(w<0.8 \%)$, 产物 $\mathrm{Al}_{2} \mathrm{O}_{3}$ 呈蓝色及白色, $\mathrm{XRD}$ 测定结果 (图 5) 表明, 此时得到的 $\mathrm{Al}_{2} \mathrm{O}_{3}$ 为无 定形. 产物经 $800{ }^{\circ} \mathrm{C}$ 焙烧 $8 \mathrm{~h}$ 后, 呈白色, 此时晶型 转为 $\gamma$ 型. 经 $1100{ }^{\circ} \mathrm{C}$ 焙烧 $2 \mathrm{~h}$ 后, 晶型转为 $\theta$ 型, 并有 $\delta$ 型存在, 同时仍能保持较好的规则大孔结 构, 表现出高的热稳定性. 经 $1200{ }^{\circ} \mathrm{C}$ 焙烧 $2 \mathrm{~h}$ 后, 
$\mathrm{Al}_{2} \mathrm{O}_{3}$ 仍为 $\theta$ 型, 但大孔孔壁完全破坏。本方法制 备的大孔 $\mathrm{Al}_{2} \mathrm{O}_{3}$ 样品比 Vaudreuil 等 ${ }^{[15]}$ 用三仲丁醇 铝制得的大孔 $\mathrm{Al}_{2} \mathrm{O}_{3}$ 的热稳定性高, 他们制备的大 孔 $\mathrm{Al}_{2} \mathrm{O}_{3}$ 在 $1000{ }^{\circ} \mathrm{C}$ 焙烧 $2 \mathrm{~h}$ 孔结构就完全破坏。

\section{References}

1 Wang, D. ; Caruso, R. A. ; Caruso, F. Chem. Mater., 2001, 13: 364

2 Imhof, A. ; Pine, D. J. Nature, 1997, 389: 948

3 Velev, O. D. ; Jede, T. A. ; Lobo, R. F. ; Lenhoff, A. M. Nature, 1997, 389: 447

4 Holland, B. T. ; Blanford, C. F. ; Do, T. ; Stein, A. Chem. Mater. 1999, 11: 795

5 Yan, H. ; Blandford, C. F. ; Holland, B. T. ; Smyrl, W. H. ; Stein, A. Chem. Mater., 2000, 12: 1134

6 Shen, Y. ; Wu, Q. Z. ; Li, Y. G. Chin. J. Catalysis, 2002, 23: 179 [沈勇, 邬泉周, 李玉光. 催化学报 (Cuihua Xuebao), 2002, 23: 179]

7 Zakhidov, A. A. ; Baughman, R. H. ; Iqbal, Z. ; Cui, C. ;
Khayrullin, L. ; Dantas, S. O. ; Marti, J. ; Ralechenko, V. G. Science, 1998, 282: 897

8 Yan, H. ; Blandford, C. F. ; Lytle, J. C. ; Carter, B. ; Smyrl, W. H. ; Stein, A. Chem. Mater., 2001, 13: 4314

9 Lei, Z. ; Li, J. ; Ke, Y. ; Zhang, Y. ; Zhang, H. ; Li, P. ; Xing, J. J. Mater. Chem., 2001, 11 (12): 2930

10 Pacewska, B. ; Keshr, M. Thermochimica Acta, 2002, 385: 73

11 Chen, Z. ; Yang, S. Q. ; Jiang, H. Y. Inorganic Chemicals Industry, 1997, 4: 10 [陈 忠, 杨松青, 蒋汉瀛. 无机盐工业 ( Wujiyan Gongye), 1997, 4: 10]

12 Chen, G. R. Encyclopaedia of Chemical Industry. Beijing: Chemical Industry Press, 1996: 966 [陈桂荣. 化工百科全书. 北 京: 化学工业出版社. 1996: 966]

13 Feng, T. L. ; Gurian, P. L. ; Healy, M. D. ; Barron, A. R. Inorg. Chem., 1990, 29: 408

14 Zhang, Y. G. ; Lei, Z. B. ; Li, J. M. ; Lu, S. M. New J. Chem. 2001, 25: 1118

15 Vaudreuil, S. ; Bousmina, M. ; Kaliaguine, S. ; Bonneviot, L. Microporous and Mesoporous Mater., 2001, 44-45: 249

\title{
A New Route to Fabricate Highly Ordered Three-dimensional Macroporous $\mathrm{Al}_{2} \mathrm{O}_{3}{ }^{*}$
}

\author{
Wu Quan-Zhou Shen Yong Li Yu-Guang
}

( School of Chemistry and Chemical Engineering, Sun Yat-Sen (Zhongshan) University, Guangzhou 510275)

\begin{abstract}
Using low-cost $\mathrm{Al}\left(\mathrm{NO}_{3}\right)_{3} \cdot 9 \mathrm{H}_{2} \mathrm{O}$ as raw material and citric acid as chelator, highly ordered three-dimensional macroporous (3DOM) $\mathrm{Al}_{2} \mathrm{O}_{3}$ with pore size of $250 \sim 350 \mathrm{~nm}$ has been successfully fabricated by a template method in which polystyrene colloidal crystal with diameter of about $600 \mathrm{~nm}$ were used as template. The TGA, TG-IR and SEM observations show that adding citric acid is in favor of forming the three-dimensional ordered structure since it can avoid forming agglomerated particles during heat treatment. Within the range of $0.5 \sim 0.8 \mathrm{~mol} \cdot \mathrm{L}^{-1}$ of the precursor concentration, the $\mathrm{Al}_{2} \mathrm{O}_{3}$ samples obtained are highly ordered three-dimensional macroporous materals, and such macroporou structure is maintained up to $1100{ }^{\circ} \mathrm{C}$, showing a high thermostability.
\end{abstract}

Keywords: Three-dimensional ordered macropore, $\quad \mathrm{Al}_{2} \mathrm{O}_{3}, \quad$ Polystyrene colloidal crystal 